\title{
A Study of Attenuation of Conducted EMI Generation in a Switching Power Supply Using Filtering Technique
}

\author{
Piyawit Sripodok ${ }^{\mathrm{a}}$, Chaiyan Jettanasen ${ }^{\mathrm{a}, *}$ \\ ${ }^{a}$ Department of Electrical Engineering, Faculty of Engineering, \\ King Mongkut's Institute of Technology Ladkrabang, Bangkok 10520, Thailand \\ *Corresponding Author: kjchaiya@kmitl.ac.th
}

\begin{abstract}
This paper studies on the attenuation of conducted electromagnetic interference (EMI) generation in a switching power supply using passive filtering technique. The switching power supply functions with the switching frequency range up $20 \mathrm{kHz}$ and mostly generates noises in low-and-high frequencies; this leads to EMI problems to electronic and/or electrical devices/equipment. To overcome the problems, electromagnetic compatibility (EMC) regulation should be applied. The EMC standard for conducted emission measurement is principally defined in the frequency range of $150 \mathrm{kHz}$ to $30 \mathrm{MHz}$. The analysis and experimental results show that by inserting a passive filter to the studied switching power supply, the conducted emission can be reduced in the specified frequency range. The obtained results will be additionally compared with an EMC standard (EN55022 class B) in order to reveal the effectiveness of the applied EMI attenuation technique. Finally, the switching power supply should apply filtering technique in development of electronic and/or electrical devices/equipment.
\end{abstract}

Keywords: attenuation technique, conducted emission, electromagnetic interference (EMI), filter, switching power supply.

\section{Introduction}

Power electronic era of rapid development, switching power supply is on the basis of the development of modern electronics and technology ${ }^{(1)}$. Switching power supply is more widely used in many industrial devices/apparatus. The applications of switching power converter are such as in the information and telecommunication industry, renewable energy conversion, electric vehicles, audio amplifiers, lighting technology, power system conditioning, energy recycling in the manufacturing industry, drives, and high power industrial applications, basic principles of operation of a power electronic circuit, basic components of the power circuit, power semiconductor switches and passive reactive elements. Today, switching power supplies comprise EMI Filter for reducing conducted EMI emission for both common mode (CM) and differential mode (DM). Theoretically, each component of EMI filter can mitigate the CM and/or DM emissions. Normally, the EMI filter is composed of common mode capacitor $\left(\mathrm{C}_{\mathrm{Y}}\right)$, differential mode capacitor $\left(\mathrm{C}_{\mathrm{X}}\right)$, common mode choke $\left(\mathrm{L}_{\mathrm{CM}}\right)$ and differential mode choke $\left(\mathrm{L}_{\mathrm{DM}}\right)$. The common and differential mode chokes of EMI filter are mainly used to limit the $\mathrm{CM}$ and DM emissions, respectively ${ }^{(2-4)}$. The required attenuation of the DM filter could be calculated by selecting the largest peak value within the relevant range $(150 \mathrm{kHz}-30 \mathrm{MHz})$ and comparing this value to the limits. The required filter attenuation is used to choose an appropriate DM filter topology and the number of connected filter stages in series. On the basis of the filter topology, the corner frequency of the filter is calculated so that the attenuation is equal to the required one added with $6 \mathrm{~dB}$ margin at the given frequency. With the corner frequency of the filter, the LC values will be determined. Since the output impedance influences on the stability of the system, the controller regulates the dc output voltage to a constant voltage resulting in a constant power load behavior at the input. This behavior could lead to instability in combination with the main EMI filter impedances. For applying filtering technique, it is based on design method relying on the assumption that DM noise is dominant in the lower frequency ranges. To assure that this assumption is 
valid, filter technique will be applied to the studied system, which is herein the switching power supply ${ }^{(5)}$.

\section{Experimental System Setup and Filtering Technique}

\subsection{Test setup for EMI measurement}

Test setup usually refers to the EMC standard, such as CISPR, EN, FCC standards. The conducted EMI emission measurement is carried out in a shielded enclosure in EMC laboratory. The conducted emission is measured from $9 \mathrm{kHz}$ to $30 \mathrm{MHz}$ according to EN55022. The measurement procedure requires EMI receiver and line impedance stabilization network (LISN) with $50 \Omega / 50 \mu \mathrm{H}$ impedance for testing. The LISN must be inserted between the equipment under test (EUT) and the ac utility line to provide specified measuring impedance for noise voltage measurement. It also serves to isolate the EUT and the measuring equipment from the utility at radio frequencies. The block diagram of EMI measurement of EUT including EMI filter is shown in Fig. 1. The setup configuration of conducted emission is illustrated in Fig. 2. The EUT in Fig. 2 refers to the switching power supply and filter in this study.

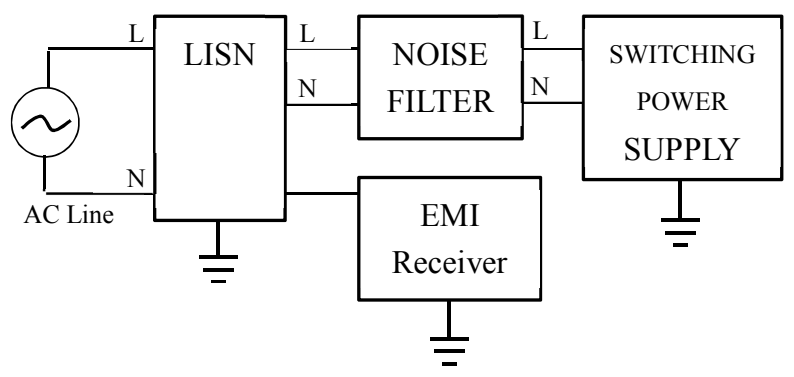

Fig. 1. Block diagram for EMI measurement of the switching power supply including EMI filter.

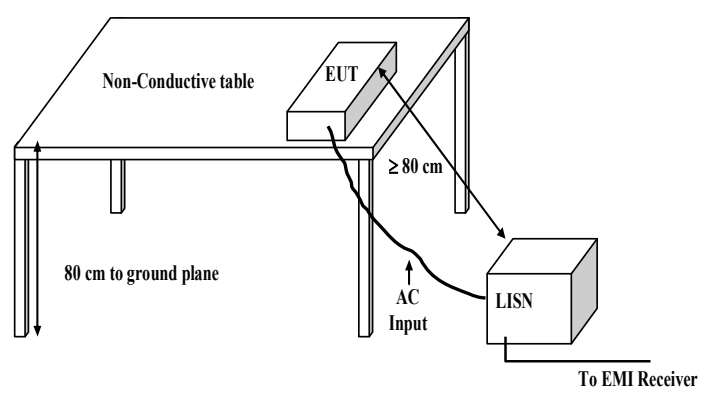

Fig. 2. Setup configuration of conducted emission measurement

\section{2 $\quad$ Filtering Technique}

Fig. 3 shows the circuit of the filter topology; common mode $(\mathrm{CM})$ and differential mode (DM) noises are dealt with different portions of the filter. The equivalent circuits of the CM and DM parts of the filter are depicted in Fig. 4 and Fig. 5, respectively. The noise voltage, measured from the $50 \Omega$ resistors contains both common-mode (CM) noise and differential-mode (DM) noise. Each mode of noise is dealt with by the respective section of an EMI filter. For Fig. 4 and Fig. 5, it is noticed that some elements of the filter affect only common-mode (CM) and differential-mode (DM) noises whereas some affect both common-mode (CM) noise and differential-mode (DM) noise. The capacitors $\mathrm{C}_{\mathrm{X} 1}$ and $\mathrm{C}_{\mathrm{X} 2}$ affect differential-mode (DM) noise only. An ideal common-mode choke $\mathrm{L}_{\mathrm{C}}$ affects common-mode (CM) noise only, but the leakage inductance $\mathrm{L}_{\text {leakage }}$ between the two windings of $\mathrm{L}_{\mathrm{C}}$ affects differential-mode $(\mathrm{DM})$ noise. $\mathrm{C}_{\mathrm{Y}}$ suppresses both common-mode (CM) noise and differential-mode (DM) noise, but its effect on differential-mode (DM) noise suppression is practically very little because of the relatively large value of $\mathrm{C}_{\mathrm{X} 2}$. Similarly, $\mathrm{L}_{\mathrm{D}}$ suppresses both differential-mode (DM) and common-mode (CM) noises, but its effect on common-mode (CM) noise is practically very little because of the relatively large value of $\mathrm{L}_{\mathrm{C}}{ }^{(6)}$.

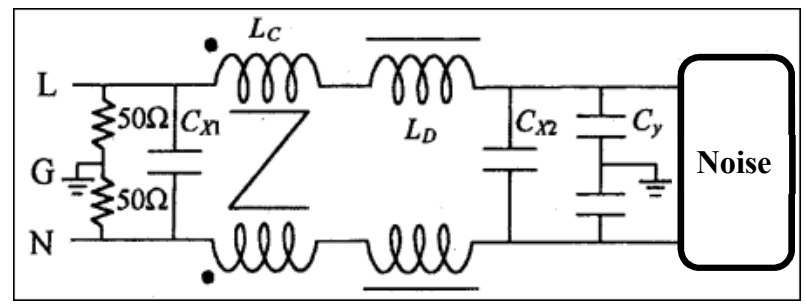

Fig. 3. Typical EMI filter topology 


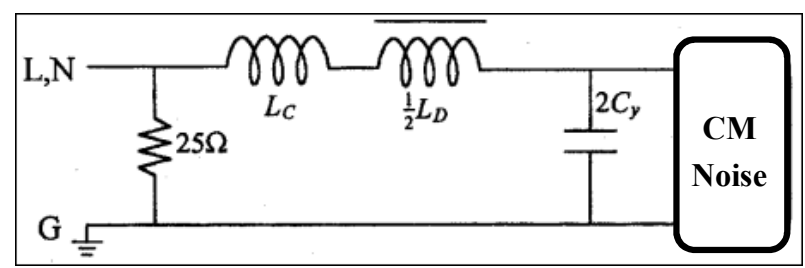

Fig. 4. Equivalent circuit of the common mode noise

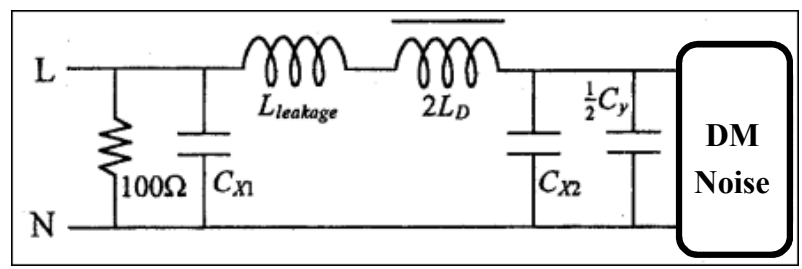

Fig. 5. Equivalent circuit of the differential mode noise

By considering Fig. 5 and calculation is based on the cut-off frequency $\left(f_{C}\right)$ of EMI filter, the selected $f_{c}$ is determined from the measured noise. The cut-off frequency is the starting frequency of signal attenuation; if one assumes that differential-mode (DM) noise series source impedance is very small, the impedance of $\mathrm{C}_{\mathrm{X} 2}$ is bigger than $\mathrm{C}_{\mathrm{Y}}$, then differential-mode (DM) attenuation is achieved. But in this case $\mathrm{L}_{\mathrm{DM}}$ is $\left(2 \mathrm{~L}_{\mathrm{D}}+\mathrm{L}_{\text {leakage }}\right)$ and $\mathrm{C}_{\mathrm{DM}}$ is $\mathrm{C}_{\mathrm{X} 1}$. So, the corner frequency for differential mode (DM) component $\mathrm{f}_{\mathrm{C}, \mathrm{DM}}$ can be determined using equation (1) ${ }^{(7)}$.

$$
f_{C, D M}=\frac{1}{2 \pi \sqrt{\left(2 L_{D}+L_{\text {leakage }}\right) \times C_{D M}}}
$$

Similarly, the EMI filter for differential-mode (DM) component can attenuation approximately $40 \mathrm{~dB} /$ decade $^{(6,8)}$.

\section{Results and Discussion}

The switching power supply; a part of radio signal receiver generates conducted noise in frequency domain as shown in Fig. 6. It shows that its switching frequency is 22 $\mathrm{kHz}$ (the first harmonic). The design method relies on the assumption that differential mode (DM) noise is dominant in the lower frequency ranges. The selected $f_{c}$ is determined from the measured noise of switching power supply. To estimate the attenuation of EMI filter, a line with slope of $40 \mathrm{~dB} /$ decade is plotted in Fig. 7. The corner frequency
$\mathrm{f}_{\mathrm{C}, \mathrm{DM}}$ for differential mode (DM) component is found to be $7.26 \mathrm{kHz}$, then substituting this value into equation (1), and choosing $\mathrm{C}_{\mathrm{X} 1}=\mathrm{C}_{\mathrm{X} 2}=0.1 \mu \mathrm{F}$, and neglecting $\mathrm{L}_{\text {leakage, thus, }}$ value of $\mathrm{L}_{\mathrm{D}}$ is found to be $2.4 \mathrm{mH}^{(6,9)}$.

$$
\begin{aligned}
7.26 \times 10^{3} & =\frac{1}{2 \pi \sqrt{\left(2 L_{D}+0\right) \times\left(0.1 \times 10^{-6}\right)}} \\
\therefore L_{D} & =2.4 m H
\end{aligned}
$$

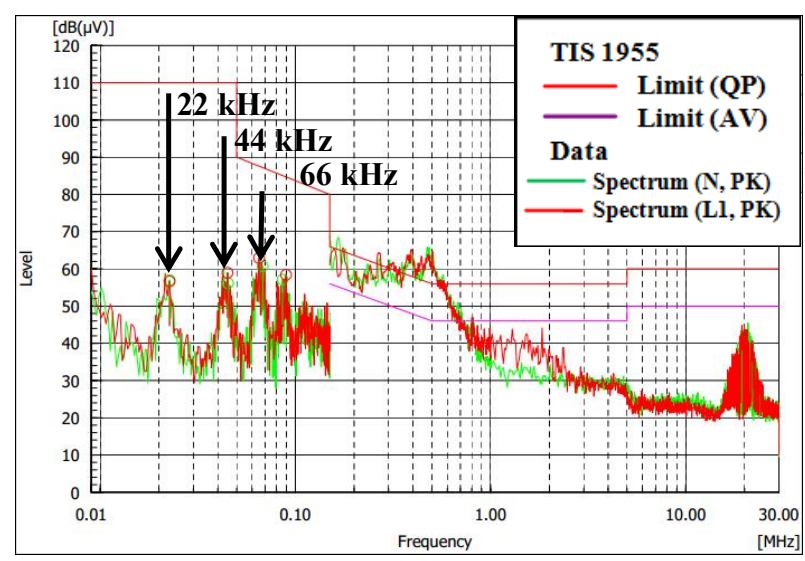

Fig. 6. Conducted noise generated by the switching power supply

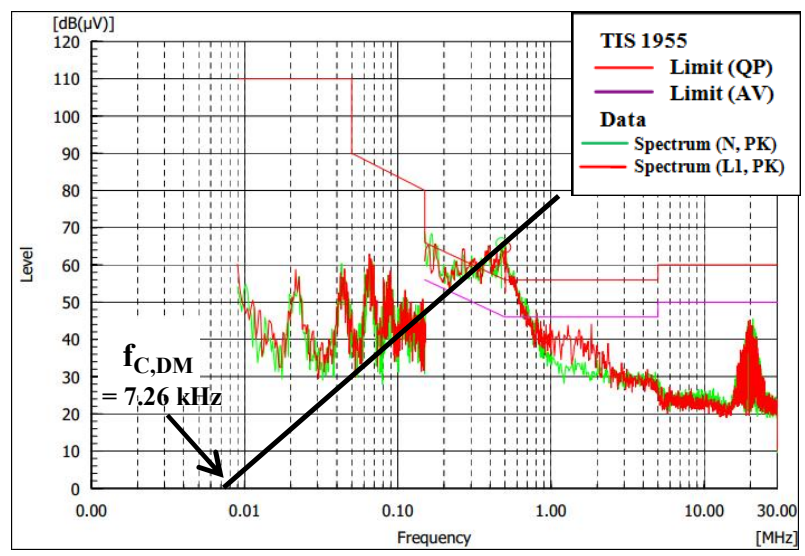

Fig. 7. Plotted corner frequency $f_{C, D M}$ for differential mode (DM) component.

According to the EMC standard, the EMI measurement of switching power supply will be carried out for both conducted emissions. The testing result of conducted noise level for only switching power supply (disconnected EMI filter) is illustrated in Fig. 8. 
Note that, conducted EMI level exceeds the standard in the frequency range of $150 \mathrm{kHz}-800 \mathrm{kHz}$.

Then, the switching power supply connecting to EMI filter is taken into account for EMI emission measurement. The testing result of conducted noise for connecting to EMI filter is illustrated in Fig. 9. The spectrum is compared with the EN55022 class B standard. It is clearly seen that the conducted noise level becomes lower than the previous case around 20 to $30 \mathrm{~dB}$ (for frequency range of $150 \mathrm{kHz}-800$ $\mathrm{kHz}$ ). This confirms the effectiveness of using filter technique for conducted EMI attenuation in switching power supply.

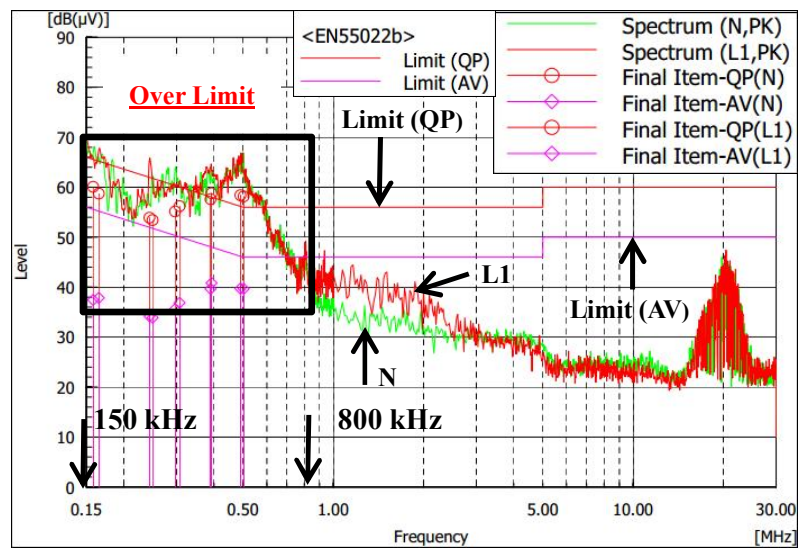

Fig. 8. Conducted noise generated by the only switching power supply (disconnected EMI filter)

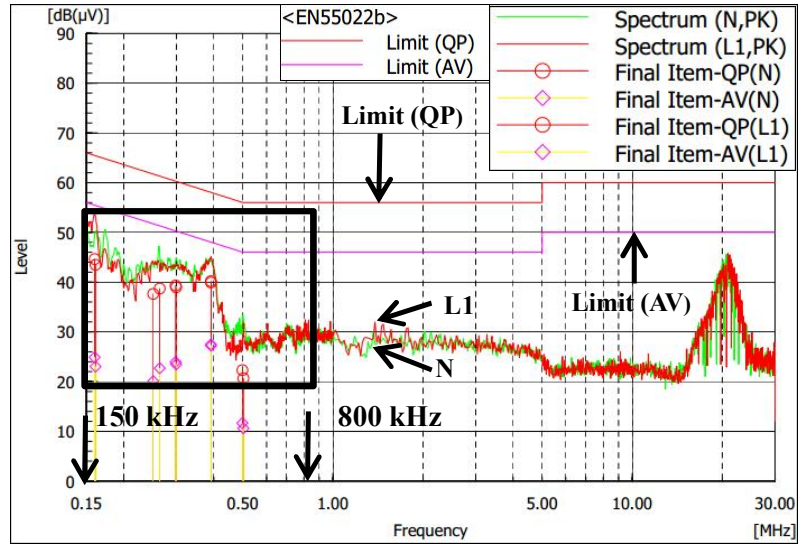

Fig. 9. Conducted noise generated by the only switching power supply (connected EMI filter)

\section{Conclusions}

The analysis and experimental results showed that the conducted noise could be attenuated by using passive filtering technique. It is found that the method developed was very promising and indicated that this method could be used for EMI studies during electronic/electrical equipment development stage for many domestic and industrial applications. Finally, the filtering technique is effective for mitigating noises issued from the switching power supply.

\section{Acknowledgment}

This work is financially supported by Faculty of Engineering Research Fund, King Mongkut's Institute of Technology Ladkrabang (KMITL), Thailand. The measurement of conducted electromagnetic interferences is supported by the Electrical and Electronic Products Testing Center (PTEC), Bangkok, Thailand. The authors would like to thank for all supports.

\section{References}

(1) Liu, Xia. "The Design and Implementation of Fly Back Type High Frequency Switching Power Supply Based on OB2201." 2015 International Conference on Intelligent Systems Research and Mechatronics Engineering. Atlantis Press, 2015.

(2) Yongbin Chu, and Shuo Wang : "A Generalized Common-Mode Current Cancelation A pproach for Power Converters", IEEE Transactions on Industrial Electronics, VOL. 62, NO. 7, July 2015.

(3) Vuttipon Tarateeraseth, Kye Yak See, Flavio G. Canavero, and Richard Weng-Yew Chang : "Systematic Electromagnetic Interference Filter Design Based on Information From In-Circuit Impedance Measurements", IEEE Transactions on Electromagnetic Compatibility, VOL. 52, NO. 3, pp.588-598, August 2010.

(4) P. Boonma, V. Tarateeraseth, and W. Khan-ngern : “A study of integrated chocke characteristic for EMI Filter Design", IC EMC Internation Conference on Electromagnetic Compatibility, Phuket 2005. 
(5) Juergen Biela, Roman Waespe, Marcello Lobo Heldwein, Klaus Raggl and Johann W. Kolar : "Passive and Active Hybrid Integrated EMI Filters", IEEE Transactions on Industrial Electronics, VOL. 24, NO. 5, pp.1340-1349, May 2009.

(6) Shih, Fu-Yuan, Yie-Tone Chen, Yan-Pei Wu, Yie-Tone Chen : "A procedure for designing EMI filters for AC line applications", IEEE Transactions on Power Electronics, VOL.11, NO.1, pp.170, 181, January 1996.

(7) Raggl, K.; Nussbaumer, T., Kolar, J.W. : "Guideline for a Simplified Differential-Mode EMI Filter Design," IEEE Transactions on Industrial Electronics, VOL.57, NO.3, pp.1031, 1040, March 2010.

(8) Baris Colak, and H. Bulent Ertan : "Design Issues and a Novel Approach for Measurement and Elimination of Conducted EMI for an Induction Cooktop”, IC EMC Internation Conference on Electromagnetic Compatibility, Phuket 2005.

(9) Yong-Wook Kim, Jun-Ho Kim, Ki-Young Choi, Bum-Seok Suh, and Rae-Young Kim : "A Novel Soft-Switched Auxiliary Resonant Circuit of a PFC ZVT-PWM Boost Converter for an Integrated Multichip Power Module Fabrication", IEEE Transactions on Industrial Applications, VOL. 49, NO. 6, pp.2802-2809, November/December 2013. 